\title{
Reference
}

1. Sebastian Feuerlein S, Pauls S, Markus S. Juchems MS, et .al, Pitfalls in Abdominal Diffusion-Weighted Imaging: How Predictive is Restricted Water Diffusion for Malignancy. AJR 2009; 193:1070-1076

Dr. Adilson Prando

Head, Department of Radiology and

Diagnostic Imaging, Vera Cruz Hospital

Campinas, São Paulo, Brazil

E-mail: adilson.prando@gmail.com

doi: $10.1590 / S 1677-553820100002000019$

\section{Split-bolus MDCT urography: Upper tract opacification and performance for upper tract tumors in patients with hematuria}

Maheshwari E, O’Malley ME, Ghai S, Staunton M, Massey C

Joint Department of Medical Imaging, University of Toronto, ON, Canada

AJR Am J Roentgenol. 2010; 194: 453-8.

Objective: Our purpose was to assess upper urinary tract opacification and the performance of split-bolus MDCT urography for upper tract tumors in patients with hematuria.

Materials and Methods: Between January 2004 and December 2006, we identified 200 patients (119 men, 81 women; median age, 58 years, age range, 18-89 years) who underwent MDCT urography for hematuria. MDCT urography included unenhanced and combined nephrographic and excretory phase imaging of the urinary tract. Images were independently reviewed by two radiologists blinded to the final diagnosis. The degree of upper urinary tract opacification and the diagnosis were recorded. Prospective interpretations were also reviewed. The standard of reference included all available clinical, imaging, and laboratory data for up to 12 months after MDCT urography. Sensitivity, specificity, accuracy, and positive and negative predictive values were calculated for upper tract tumors for prospective and retrospective interpretations.

Results: For reviewers 1 and 2, 85.1\% and $84.5 \%$ of segments were at least $50 \%$ opacified, respectively. Final diagnoses for hematuria were no cause, 123 (61.5\%); urothelial cancer, 27 (13.5\%); nonmalignant, 46 (23\%) and indeterminate, four patients (2\%). There were nine upper tract cancers. Sensitivity, specificity, and accuracy for upper tract cancers for prospective interpretation, reviewer 1 and reviewer 2 , were $100 \%, 99 \%, 99 \% ; 100 \%$, $99.5 \%, 99.5 \%$; and $88.9 \%, 99.0 \%, 98.5 \%$, respectively.

Conclusion: Split-bolus MDCT urography provided at least 50\% opacification of the majority of upper urinary tract segments and had high sensitivity, specificity, and accuracy for the detection of upper urinary tract tumors.

\section{Editorial Comment}

Multidetector CT-urography (MDCT-urography) has been shown to be an effective single comprehensive examination in the evaluation of patients with hematuria or with risk for the development of urothelial malignancies. Since protocols for MDCT urography varies from each institution, most MDCT-urography images are obtained in the unenhanced phase (detection of calculi), nephrographic-phase (detection of renal masses) and excretory-phase (detection of urothelial lesions). The authors present their results with a protocol called split-bolus MDCT- urography where the unenhanced phase is followed only by a combined nephrographic and excretory phase. During split-bolus, CT-urography the intravenous injection of contrast material is performed in two steps. First, $40 \mathrm{ml}$ is injected at $2 \mathrm{ml} / \mathrm{s}$ and after 120 second from the beginning of the first injection, the 
remaining $80 \mathrm{ml}$ is injected. According the authors in patients with hematuria, split-bolus MDCT- urography and oral hydration provide complete opacification of the majority of upper urinary tract segments and are accurate for the diagnosis of upper tract urothelial tumors. Since the main objective of MDCT-urography is to detect all possible causes of hematuria, this study has some limitations. The authors did no include an analysis of the capability of split-bolus technique for the detection of urinary calculi, renal parenchymal tumor and bladder cancers. As we know small bladder cancer can be missed if only excretory phase of the full bladder is obtained.

Another issue that could be addressed is how the renal parenchymal masses can be adequately characterized by the combined nephrographic /excretory phase obtained with split-bolus technique. Classically, renal masses are best characterized by the combination of findings obtained without intravenous contrast enhancement, scans obtained in nephrographic phase (70-90") and scans obtained in the excretory phase. In our opinion split bolus MDCT- urography may be useful for follow up patients with higher risk of develop upper tract urothelial cancer, particularly those already evaluated with cistoscopy. These patients should benefit with the use of this examination, which has high accuracy for the detection of urothelial cancer and uses low dose of radiation.

Dr. Adilson Prando

Head, Department of Radiology and

Diagnostic Imaging, Vera Cruz Hospital

Campinas, São Paulo, Brazil

E-mail: adilson.prando@gmail.com

\section{PATHOLOGY}

doi: $10.1590 /$ S1677-553820100002000020

\section{Should pathologists continue to use the current pT2 substaging system for reporting of radical prostatectomy specimens?}

Billis A, Meirelles L, Freitas LL, Magna LA, Ferreira U

Department of Anatomic Pathology, School of Medicine, University of Campinas, Unicamp, Brazil

Mod Pathol. 2010; 23 (suppl 1): 179A

Background: During the International Society of Urological Pathology (ISUP) consensus conference on handling and staging of radical prostatectomy specimens, $65.5 \%$ of the attendants answered that the current pT2 susbstaging system should not be used. Answering to another question, $63.4 \%$ favored to be reduced to two categories based on studies showing that $\mathrm{pT} 2 \mathrm{~b}$ does not exist. There was no consensus in regard to a minimum size for a second tumor to be considered for the whole case to be classified as pT2c as well as in regard to the definition of index tumor. We compared clinicopathologic findings and biochemical progression following surgery classifying pT2 patients into two categories.

Design: The study was based on whole-mount consecutive surgical specimens from 142 patients with organ confined cancer. Using a semiquantitative method for evaluation of tumor extent, 10 positive points corresponds roughly to a $0.5 \mathrm{ml}$ tumor. We considered $\mathrm{pT} 2 \mathrm{a} / \mathrm{pT} 2 \mathrm{~b}$ substage (group 1 ) whenever a tumor presented $>10$ positive points on only one side and pT2c whenever presented $>10$ positive points on each of right and left side (group 2). The variables analyzed were: age, preoperative PSA, clinical stage, Gleason score on needle biopsy, and biochemical progression following surgery defined as PSA $>0.2 \mathrm{ng} / \mathrm{mL}$. The data were analyzed using 